\title{
On The Testing of the Magnetic Field Integral Equation With RWG Basis Functions in Method of Moments
}

\author{
Juan M. Rius, Member, IEEE, Eduard Úbeda, and Josep Parrón
}

\begin{abstract}
For electromagnetic analysis using Method of Moments (MoM), three-dimensional (3-D) arbitrary conducting surfaces are often discretized in Rao, Wilton and Glisson basis functions. The MoM Galerkin discretization of the magnetic field integral equation (MFIE) includes a factor $\Omega_{0}$ equal to the solid angle external to the surface at the testing points, which is $2 \pi$ everywhere on the surface of the object, except at edges or tips that constitute a set of zero measure. However, the standard formulation of the MFIE with $\Omega_{0}=2 \pi$ leads to inaccurate results for electrically small sharp-edged objects. This paper presents a correction to the $\Omega_{0}$ factor that, using Galerkin testing in the MFIE, gives accuracy comparable to the electric field integral equation (EFIE), which behaves very well for small sharp-edged objects and can be taken as a reference.
\end{abstract}

Index Terms-Electromagnetic scattering, integral equations, numerical analysis, radar cross sections.

\section{INTRODUCTION}

$\mathbf{E}$ LECTRIC and magnetic field integral equations [2] are widely used in conjunction with Method of Moments (MoM) discretization [1] for the numerical analysis of electromagnetic radiation and scattering. The MoM Galerkin discretization of the electric field integral equation (EFIE) for three-dimensional (3-D) arbitrary surfaces was presented by Rao, Wilton and Glisson (RWG) in the well-known reference [3]. The linear triangle basis function presented in [3] will be denoted here as RWG basis function. An analogous formulation for the magnetic field integral equation (MFIE) for 3-D arbitrary surfaces was later developed [4].

As will be shown later, the MFIE formulation includes a factor $\Omega_{0}$, equal to the solid angle external to the surface at the field evaluation point. Since the Galerkin testing integration points are placed inside triangles, $\Omega_{0}$ is equal to $2 \pi$ at all the testing points. The resulting formulation provides excellent results for smooth geometries [4]. However, for small sharp-edged objects, a significant error appears in the MFIE when compared to the MoM-EFIE. The last can be taken as a reference since it is known to work well for those objects. The

\footnotetext{
Manuscript received August 29, 2000; revised January 9, 2001. This work was partially supported by the Spanish Comisión Interministerial de Ciencia y Tecnología (CICYT) under Project TIC 98-1037. E. Úbeda and J. Parrón were supported by the Generalitat de Catalunya, Comissionat per a Universitats i Recerca, under Grants 1997 FI 00747 and 1997 FI 00679.

The authors are with the Department Teoria del Senyal i Comunicacions (TSC), Universitat Politècnica de Catalunya, 08034 Barcelona, Spain.

Publisher Item Identifier S 0018-926X(01)07641-4.
}

aim of this paper is to decrease the error of the MoM-MFIE for small sharp-edged objects. Although this error would be smaller with a better choice of the testing functions set [6], [7], we will restrict our attention here to Gakerkin testing functions.

For sharp-edged objects of electrically small dimensions, the contribution of basis functions located at edges is particularly important, since the boundary of many basis functions is on object edges. The fact that at tips and edges the solid angle external to the surface is clearly different than $2 \pi$ suggests us that a better value of $\Omega_{0}$ could be used for the testing points on triangles neighbor to edges. On the other hand, for electrically larger sharp-cornered objects; 1) diffraction at edges is less important than reflection at surfaces and 2) the number of basis functions neighbor to edges is relatively smaller and as a consequence, the sensibility of the scattering computations to the solid angle choice is unnoticeable.

In this paper attention is limited to sharp-edged objects of small electrical dimensions. The aim is to develop a correction to the standard version of the MoM-MFIE by modifying the value of the solid angle in order to obtain results similar to the MoM-EFIE ones. Although the improvement will be shown here only for objects of small electrical dimensions, the validity of the correction is extendable to all kinds of surfaces, including electrically large either sharp-edged or smooth-varying ones. In these cases, since the results from the standard MoM-MFIE were already good, the correction turns out imperceptible.

\section{FORMULATION: EFIE AND MFIE}

Let us consider an arbitrarily shaped 3-D closed conducting object, with its surface discretized in nonoverlapping triangles. For RCS computations, the incident field in the scattering problem is an impinging plane wave. The induced current $\vec{J}$ is expanded in RWG basis functions $\vec{f}_{n}[3]$ as

$$
\vec{J}=\sum_{n=1}^{N} \vec{f}_{n}\left(\vec{r}^{\prime}\right) I_{n}
$$

where $I_{n}$ are the unknown current coefficients to be determined in the solution of the problem.

The EFIE in a perfect electric conductor (PEC) surface forces the electric field boundary condition

$$
\left.\vec{E}\right|_{\tan }=\left.\vec{E}^{s}\right|_{\tan }+\left.\vec{E}^{i}\right|_{\tan }=0
$$


where $\vec{E}^{i}$ is the incident field and $\vec{E}^{s}$ is the scatterered field, that is, the field due to the induced currents

$$
\begin{aligned}
\vec{E}^{s}= & -j k \eta \int_{S^{\prime}} \vec{J}\left(\vec{r}^{\prime}\right) G\left(\vec{r}-\vec{r}^{\prime}\right) d S^{\prime} \\
& +j \frac{\eta}{k} \int_{S^{\prime}} \nabla^{\prime} \cdot \vec{J}\left(\vec{r}^{\prime}\right) \nabla^{\prime} G\left(\vec{r}-\vec{r}^{\prime}\right) d S^{\prime}
\end{aligned}
$$

where $G$ denotes the free-space Green's function and the time dependence $e^{j \omega t}$ is implicit.

The EFIE (2) is discretized using MoM [1] as a system of linear equations that can be expressed in matrix form as [3]

$$
\left[Z_{m n}^{E}\right]\left[I_{n}\right]=-\left[E_{m}^{i}\right] \quad n, m=1 \cdots N
$$

where $Z_{m n}^{E}=\left\langle\vec{f}_{m}, \vec{E}^{S}\left(\vec{f}_{n}\right)\right\rangle, E_{m}^{i}=\left\langle\vec{f}_{m}, \vec{E}^{i}\right\rangle$. Here $\langle\vec{f}, \vec{g}\rangle$ denotes the Hilbert inner product $\int \vec{f}\left(\vec{r}^{\prime}\right)^{*} . \vec{g}\left(\vec{r}^{\prime}\right) d S^{\prime}$.

Similarly, the MFIE is derived from the magnetic field boundary condition

$$
\hat{n} \times \vec{H}=\vec{J} \Rightarrow \hat{n} \times \vec{H}^{s}-\vec{J}=-\hat{n} \times \vec{H}^{i}
$$

where $\hat{n}$ denotes the unit normal vector to surface. Again, $\vec{H}^{i}$ refers to the incident field and the scattered field $\vec{H}^{S}$ is due to the induced currents

$$
\vec{H}^{S}=\int_{S^{\prime}} \vec{J}\left(\vec{r}^{\prime}\right) \times \nabla^{\prime} G\left(\vec{r}-\vec{r}^{\prime}\right) d S^{\prime}
$$

For observation points on the surface of the scatterer, the surface integration in (6) involves an improper integral that must be split into two definite parts [2]

$$
\hat{n} \times \vec{H}^{S}(\vec{r})=\int_{P V} \hat{n} \times\left(\vec{J} \times \nabla^{\prime} G\right) d S^{\prime}+\frac{\Omega_{0}(\vec{r})}{4 \pi} J(\vec{r})
$$

where $P V$ denotes the Cauchy principal value integration and $\Omega_{0}(\vec{r})$ stands for the solid angle external to the surface, as depicted in Fig. 1.

The need for correction in (7) is not apparent here, since $\Omega_{0}(\vec{r})=2 \pi$ everywhere on the conducting object except at an edge or a tip. Edges and tips have no surface area and therefore they do not contribute to the surface integral that arises in the testing procedure. In addition, although the solid angle external to an edge or a tip is well defined, the validity of (7) is questionable at an edge or a tip because the current $\vec{J}(\vec{r})$ could be ill behaved and even if $\vec{J}(\vec{r})$ was well behaved, the quantity $\Omega_{0}(\vec{r}) \vec{J}(\vec{r})$ would be still difficult to evaluate [6].

After the MoM discretization of (5) and (6), allowing for [4] and defining $D_{m n}=\left\langle\vec{f}_{m}, \vec{f}_{n}\right\rangle, Z_{m n}^{H}=\left\langle\vec{f}_{m}, \hat{n} \times \vec{H}^{S}\left(\vec{f}_{n}\right)\right\rangle$ and $H_{m}^{i}=\left\langle\vec{f}_{m}, \hat{n} \times \vec{H}^{i}\right\rangle$, the MFIE (5) becomes

$$
\begin{aligned}
& {\left[Z_{m n}^{H}\right]\left[I_{n}\right]-\left[D_{m n}\right]\left[I_{n}\right]=-\left[H_{m}^{i}\right]} \\
& {\left.\left[Z_{m n}^{H}\right]\right|_{P V}\left[I_{n}\right]+\frac{\Omega_{0}}{4 \pi}\left[D_{m n}\right]\left[I_{n}\right]-\left[D_{m n}\right]\left[I_{n}\right]} \\
& \quad=-\left[H_{m}^{i}\right] \quad n, m=1 \cdots N \\
& {\left.\left[Z_{m n}^{H}\right]\right|_{P V}\left[I_{n}\right]+\left(\frac{\Omega_{0}}{4 \pi}-1\right)\left[D_{m n}\right]\left[I_{n}\right]=-\left[H_{m}^{i}\right] .}
\end{aligned}
$$

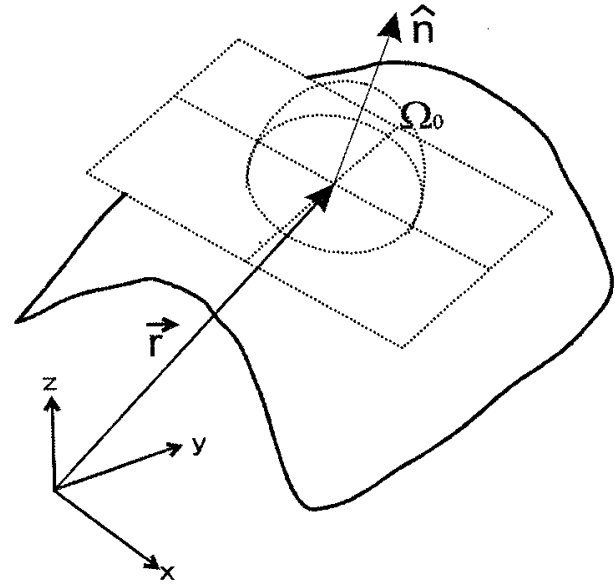

Fig. 1. Local solid angle.

The assumption $\Omega_{0}(\vec{r})=2 \pi$ is often made and (8) is simplified to the standard MFIE [4]

$$
\left.\left[Z_{m n}^{H}\right]\right|_{P V}\left[I_{n}\right]-\frac{1}{2}\left[D_{m n}\right]\left[I_{n}\right]=-\left[H_{m}^{i}\right], \quad n, m=1 \cdots N .
$$

\section{COMPUTATION}

\section{A. Source Integration}

In order to achieve the maximum accuracy in source integrals, the singular terms of both operators $(1 / R$ for EFIE and $1 / R, 1 / R^{2}$ for MFIE) are analytically integrated following [4] and [5]. After extraction of the singular terms, the remaining ones are slow-varying and can be numerically computed considering a four point quadrature rule on each triangle. Furthermore, in the MoM-MFIE formulation the cross-product can be moved out of the integral as suggested in [4]. This accurate computation is very important when analyzing electrically small geometries in order to ensure unbiased values for $\vec{H}^{S}$ and $\vec{E}^{S}$.

\section{B. Field Integration}

Whenever the field and source triangles are the same, the Galerkin testing integrals have been carried out through a fourpoint Gauss quadrature rule. When the field and source triangles are different, either four or one points are used in the quadrature summation for all triangle pairs, leading to two sets of results of different accuracy.

Figs. 3-7, respectively, show the bistatic RCS $\left(\sigma / \lambda^{2}\right)$ of a cube with side of $0.1 \lambda$ and 48 facets, a cube with side of $0.2 \lambda$ and 48 facets, a rectangular basis pyramid with side $0.07 \lambda$ and 72 facets, a regular octahedron with side $0.07 \lambda$ and 72 facets, and a cone with basis radius of $0.05 \lambda$, height of $0.04 \lambda$ and 288 facets. In Fig. 7, the cone is overdiscretized along the polar direction in order to cancel the influence of a coarse discretization of the curvature. In all cases the impinging plane wave has $+z$ axial incidence and $x$-polarized electric field.

One remarks on the disagreement of the MoM-MFIE results with the MoM-EFIE ones despite the imposed high accuracy for the computed scattered fields. Since the conditioning of the linear systems (4) and (8) is good, the only reasons for the disagreement must be; 1) the inaccuracy of testing integrals and 2) the improper choice of $\Omega_{0}$ in the MFIE. The MoM-EFIE is very 


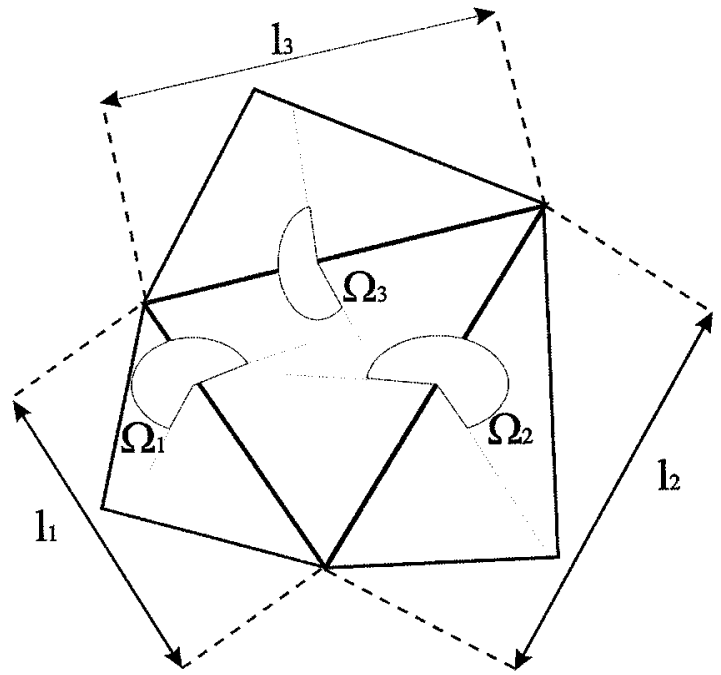

Fig. 2. Local solid angle estimate.

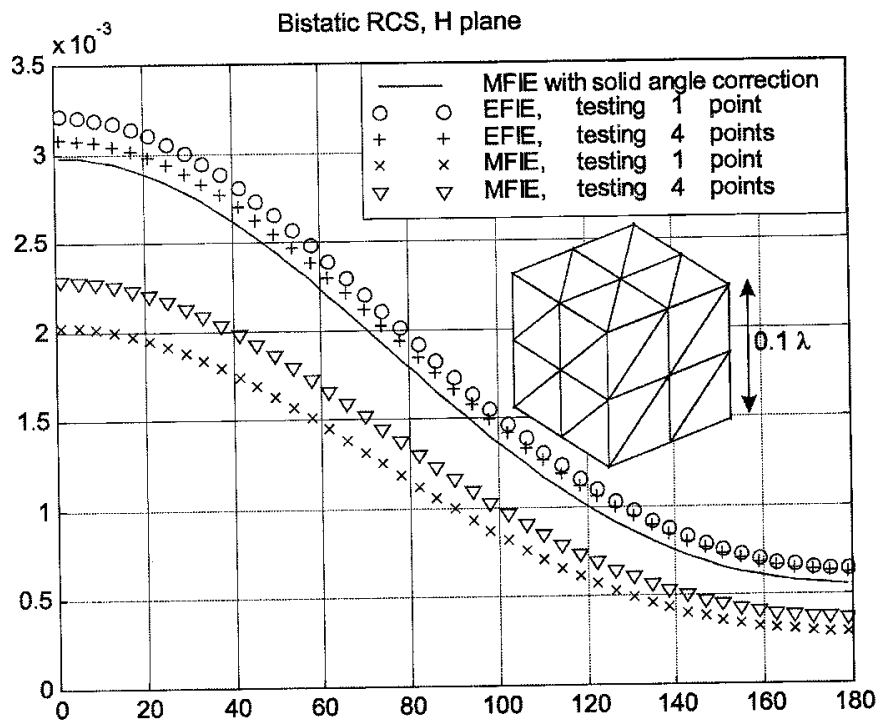

Fig. 3. $H$ plane bistatic RCS for a perfectly conducting cube with side of $0.1 \lambda$.

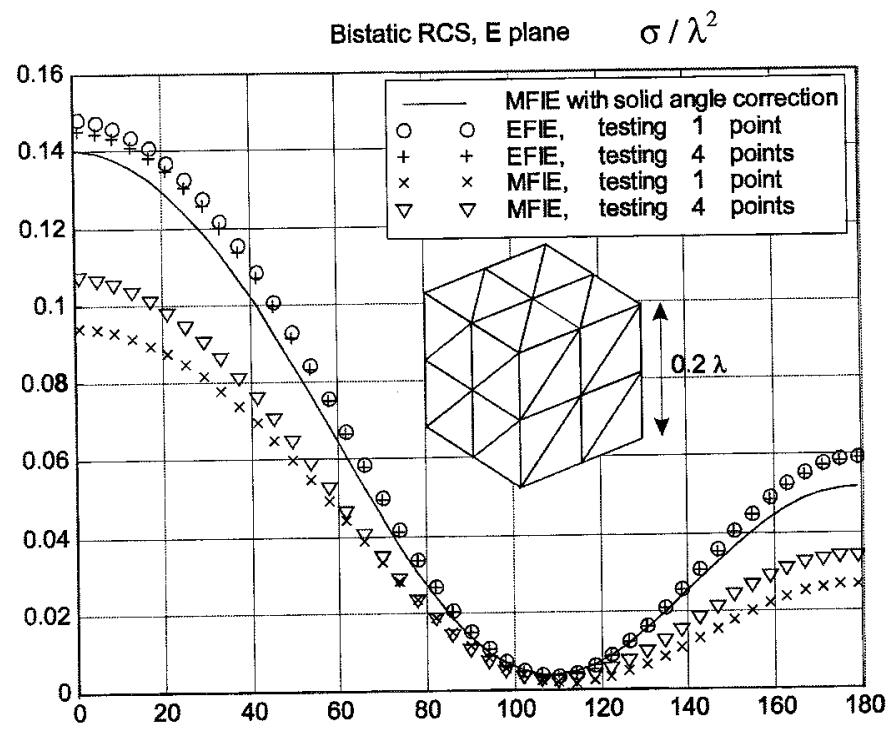

Fig. 4. $E$ plane bistatic RCS for a perfectly conducting cube with side of $0.2 \lambda$.

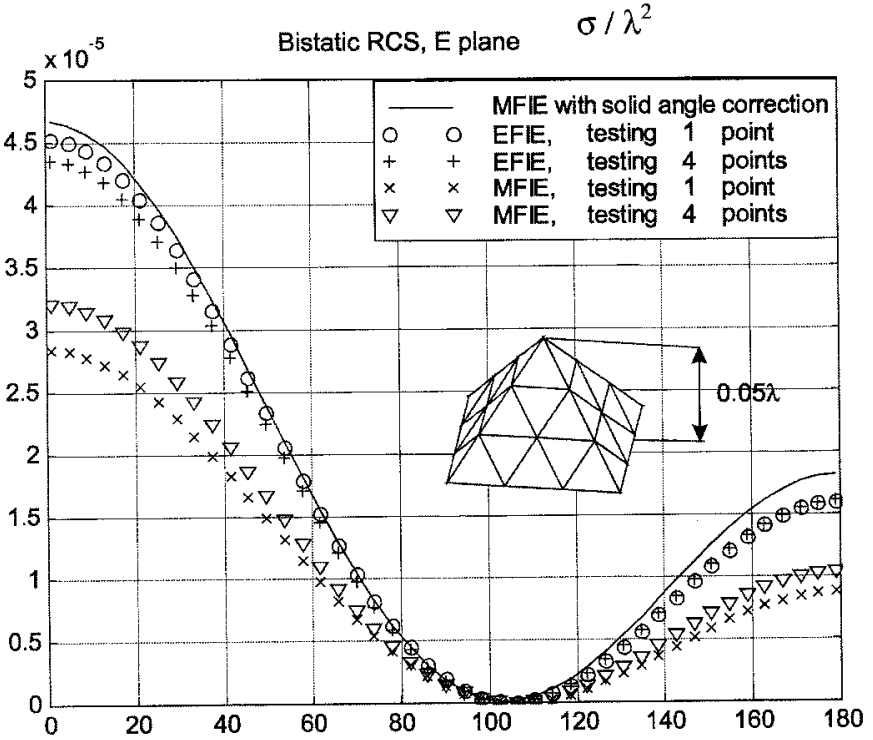

Fig. 5. $\quad E$ plane bistatic RCS for a perfectly conducting pyramid with side of $0.07 \lambda$.

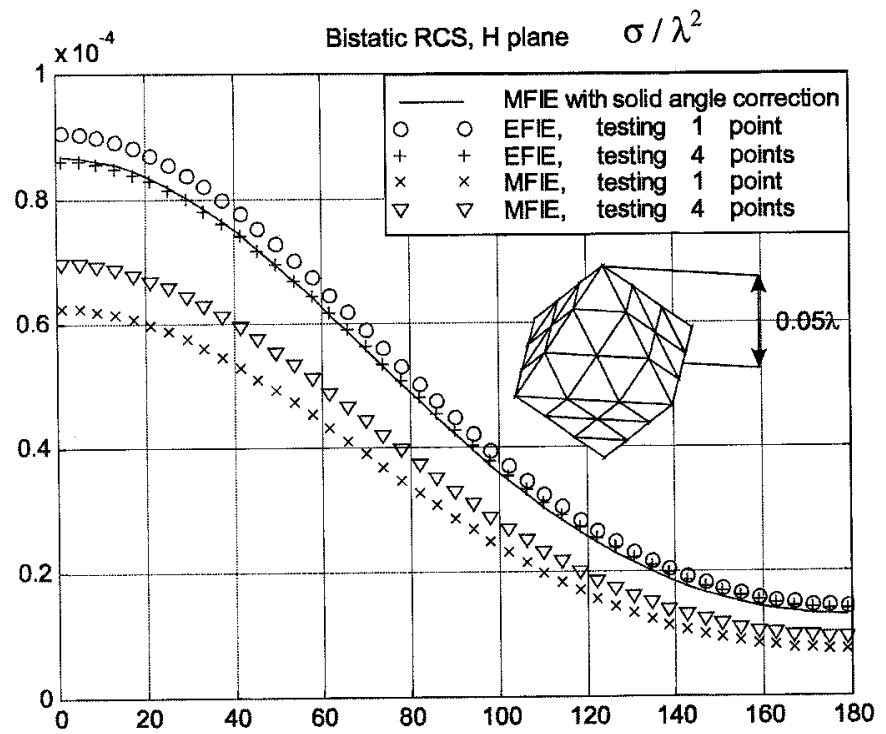

Fig. 6. $\quad H$ plane bistatic RCS for a perfectly conducting octahedron with side of $0.07 \lambda$.

robust since its results are very similar when varying the number of field testing integration points and, furthermore, there is no solid angle $\Omega_{0}$ in the EFIE formulation.

On the other hand, the MoM-MFIE results show a significant dependence on the number of field testing integration points. Indeed, when increasing the order of the Gauss quadrature rule, the MoM-MFIE results approach slowly to the MoM-EFIE reference, but still an important error remains.

\section{MOM-MFIE CORRECTION}

In order to reduce the MoM-MFIE error, we propose a correction on the MoM-MFIE operator by slightly modifying the solid angle value. By trial and error, we have been able to define the new equivalent solid angle value any triangle as the weighted 


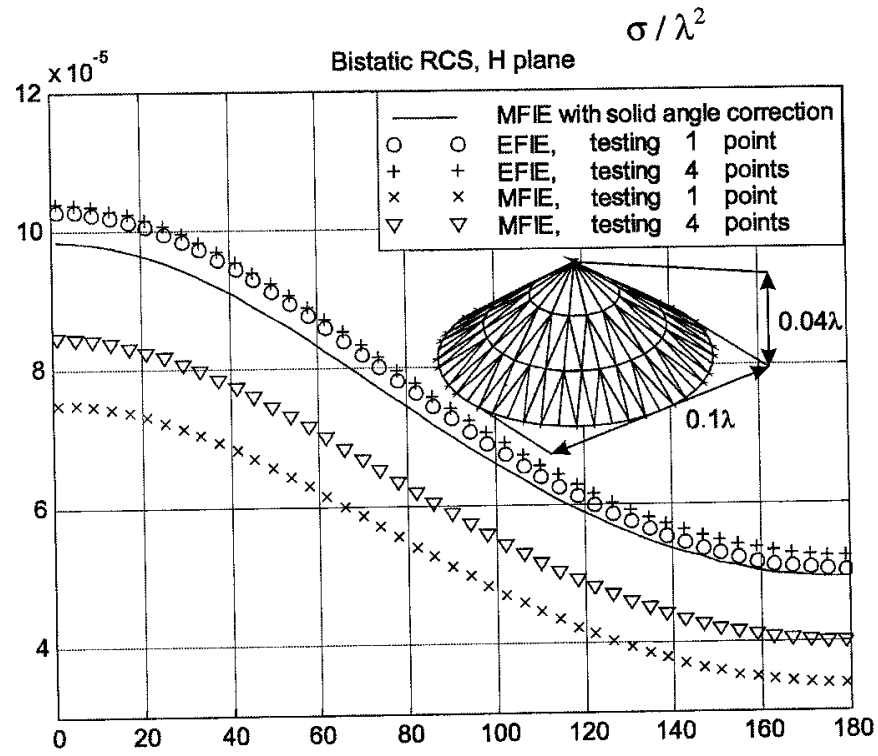

Fig. 7. $H$-plane bistatic RCS for a perfectly conducting cone with basis radius of $0.05 \lambda$ and height of $0.04 \lambda$.

average of the local solid angles over each edge of the triangle (Fig. 2)

$$
\Omega_{e q}=\frac{\left(l_{1} \Omega_{1}+l_{2} \Omega_{2}+l_{3} \Omega_{3}\right)}{\left(l_{1}+l_{2}+l_{3}\right)}
$$

where $l_{1}, l_{2}$ and $l_{3}$ are the lengths of the triangle edges.

One can readily obtain the new operator by replacing $\Omega_{0}$ by $\Omega_{e q}$ in (8). This correction approaches the MoM-MFIE results to the MoM-EFIE ones for any geometry.

The modified MoM-MFIE operator is very accurate for all the geometries tested as long as one quadrature integration point is used in MoM Galerkin testing. One can assess its good behavior for sharp-edged geometries in Figs. 3-7. The accuracy is comparable to the MoM-EFIE with four quadrature points, but the modified MoM-MFIE excels thanks to its simplicity and fast computation, since only one quadrature integration point is required.

\section{CONCLUSION}

The behavior of the MFIE and the EFIE for the analysis of electrically small sharp-edged objects has been studied. The inaccuracy of the MFIE for this kind of objects is assumed to be caused by an inappropriate choice of the external solid angle value at field evaluation points inside triangles neighbor to edges. In this paper, we propose a correction for the solid angle value that allows for the external solid angles at triangle edges. The resulting approach needs only one field evaluation point at each triangle and is as accurate as the EFIE with four quadrature integration points in Galerkin testing.

\section{REFERENCES}

[1] R. F. Harrington, Field Computation by Moment Methods. New York: MacMillan, 1968
[2] N. Morita, N. Kumagai, and J. R. Mautz, Integral Equation Methods for Electromagnetics. Norwood, MA: Artech House, 1990.

[3] S. M. Rao, D. R. Wilton, and A. W. Glisson, "Electromagnetic scattering by surfaces of arbitrary shape," IEEE Trans. Antennas Propagat., vol. AP-30, pp. 409-418, May 1982.

[4] R. E. Hodges and Y. Rahmat-Samii, "The evaluation of MFIE integrals with the use of vector triangle basis functions," Microwave Opt. Technol. Lett., vol. 14, no. 1, pp. 9-14, January 1997.

[5] D. R. Wilton, S. M. Rao, A. W. Glisson, D. H. Schaubert, O. M. Al-Bundak, and C. M. Butler, "Potential integrals for uniform and linear source distributions on polygonal and polyhedral domains," IEEE Trans Antennas Propagat., vol. AP-32, pp. 276-281, Mar. 1984.

[6] Reviewer's comments.

[7] E. Úbeda, "Contribution to the improvement of integral equation methods for penetrable scatterers," Ph.D. dissertation, Universitat Politècnica de Catalunya, Jan. 2001.

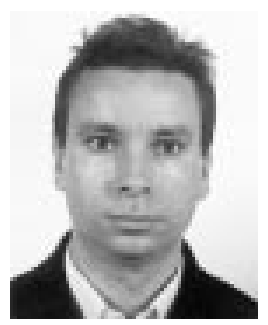

Juan M. Rius (M'89) received the Ingeniero de Telecomunicación degree and the Doctor Ingeniero degree from Politechnic University of Catalunya (UPC), Barcelona, Spain in 1987 and 1991, respectively.

In 1985, he joined the Electromagnetic and Photonic Engineering group at UPC, where he currently is Associate Professor. Since 1989, he has been engaged in the research for new and efficient methods for numerical computation of electromagnetic scattering and antenna radiation. $\mathrm{He}$ developed the Graphical Electromagnetic Computation (GRECO) approach for high-frequency RCS computation, the Integral Equation formulation of the Measured Equation of Invariance (IE-MEI) and the Multilevel Matrix Decomposition Algorithm in 3D (MLMDA-3D). He has been a Visiting Professor and CLUSTER Chair at EPFL (Lausanne); and a Visiting Fellow at City University of Hong Kong, China. He has 25 publications in refereed international journal papers and 55 in international conferences.

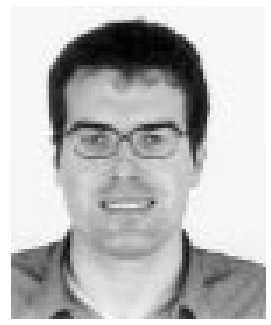

Eduard Úbeda was born in Barcelona, Spain, in 1971. He received the Telecommunication Engineer degree and the Doctor Ingeniero degree from the Politechnic University of Catalunya (UPC), Barcelona, Spain, in 1995 and 2001, respectively.

In 1996, he was with the European Commission Joint Research Center, Ispra, Italy. From 1997 to 2000, he was a Research Assistant at the Electromagnetic and Photonic Engineering group at UPC. Since 2001, he has been with the Electrical Engineering Department, Pennsylvania State University, University Park. He is author of three papers in international journals and 11 in international conference proceedings. His main research interests include numerical computation of scattering and radiation using integral equations.

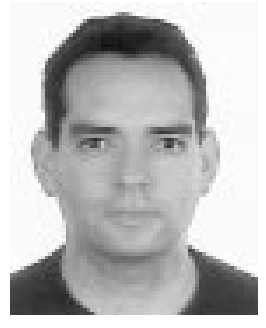

Josep Parrón was born in Sabadell (Barcelona), Spain in 1970. He received the telecommunication engineer degree from the Politechnic University of Barcelona, Spain, in 1994.

In 1997, he became a Ph.D. student in the Signal Theory and Communication Department, Politechnic University of Barcelona, and since 2000, he has been an Assistant Professor. He is author of five papers in international journals and 12 in international conference proceedings. His main research interests include fast algorithms for computational electromagnetics and their application to fractal antennas, multilayered mediums, and superconductors. 